\title{
Myomectomy as a Pregnancy-Preserving Option in the Carefully Selected Patient
}

\author{
U. Hasbargen ${ }^{a} \quad$ A. Strauss ${ }^{a} \quad$ M. Summerer-Moustaki ${ }^{a} \quad$ G. Baretton ${ }^{b}$ \\ U. Roth ${ }^{c}$ R. Kimmig a H. Heppa \\ Departments of a Obstetrics and Gynecology, ${ }^{\mathrm{b} P a t h o l o g y, ~ a n d ~}{ }^{\mathrm{c} A n e s t h e s i o l o g y, ~ K l i n i k u m ~ G r o s s h a d e r n, ~}$ \\ Ludwig Maximilians University, Munich, Germany
}

\section{Key Words}

Pregnancy · Myomectomy · Borderline lesion · Fetal limb defect

\begin{abstract}
Objectives: To present the indications for myomectomy during pregnancy and to discuss complications possibly related and unrelated to the procedure. Method and Results: A 33-year-old patient at 18 weeks of gestation underwent removal of a 1,570-gram symptomatic fundic myoma. Histologically the patient had a leiomyomatous neoplasm of uncertain malignant potential. The pregnancy was continued under sequential observation with magnetic resonance imaging and ultrasound. At 36 weeks of gestation a healthy girl with an upper extremity limb defect was born via cesarean section. Follow-up of the mother and the child was uneventful. Conclusions: Certain known risk factors in pregnant women with myomas can predispose to complications during pregnancy. Women with such risk factors or women who have failed medical therapy should be offered the option of undergoing myomectomy as a pregnancy-preserving procedure.
\end{abstract}

\section{KARGER}

Fax + 41613061234

E-Mail karger@karger.ch

www. karger.com
(C) 2002 S. Karger AG, Basel

1015-3837/02/0172-0101\$18.50/0

Accessible online at:

www. karger.com/journals/fdt

\section{Introduction}

Uterine myomas are found in approximately $2 \%$ of pregnant women [1] and usually remain asymptomatic during pregnancy. In approximately $10 \%$ of patients, complications include increased frequency of spontaneous abortion, placental abruption, premature rupture of membranes, premature labor, postpartum hemorrhage, and postpartum sepsis [2]. The clinical management of uterine myomas during pregnancy is primarily medical, but in cases of failed medical treatment, surgical intervention and myomectomy may be the only therapeutic option.

We report the case of a primipara in whom a 1,570gram myoma was removed at 18 weeks of gestation, with minimal morbidity and no need for blood transfusion.

\section{Case Report}

A 33-year-old gravida II, para I was referred to our antenatal clinic at 18 weeks of gestation, complaining of abdominal fullness and right upper quadrant discomfort, resulting from a large fundal tumor, reaching almost to the sternum. Ultrasonography showed a normal fetus, several myomas at the posterior wall of the uterus, and one fundic myoma measuring $14.0 \times 10.9 \times 10.7 \mathrm{~cm}$. The referring
Dr. Uwe Hasbargen

Klinik und Poliklinik für Frauenheilkunde und Geburtshilfe, Klinikum Grosshadern Marchioninistrasse 15, D-81377 München (Germany)

Tel +49897095 4540, Fax +49897095754

E-Mail hasbargen@gyn.med.uni-muenchen.de 
physician reported a $6-\mathrm{cm}$ growth during the prior 4 weeks. Magnetic resonance imaging (MRI) revealed a large uterus showing degenerative changes and confirmed the fundal myoma measuring $15 \mathrm{~cm}$ in diameter. The patient was aware of having myomas, but had never been advised to undergo a myomectomy prior to becoming pregnant. Her first pregnancy had resulted in a spontaneous abortion at 8 weeks of gestation.

Due to the size and rapid growth of the myoma, the patient was counseled and agreed to undergo myomectomy. A subserous fundal myoma $(14.5 \times 14.5 \times 13.0 \mathrm{~cm} ; 1,570 \mathrm{~g})$ was removed under general anesthesia. Secondary to the intramural component of the myoma, the uterine cavity was opened, exposing the dorsal plane of the fundal placenta, but keeping the amniotic membranes intact. Acute perioperative normovolemic hemodilution of $1,100 \mathrm{~cm}^{3}$ of blood and intraoperative blood salvage were employed $\left(2,259 \mathrm{~cm}^{3}\right.$ processed, $1,023 \mathrm{~cm}^{3}$ retransfused; preoperative hematocrit $35.3 \%$, postoperative hematocrit $21.7 \%$ ). No packed red blood cells were given. Several additional fibroids were visualized in the posterior wall of the uterus, but due to the blood loss and in order to avoid a second transmural defect, it was decided not to remove these myomas. On histopathological examination, a leiomyomatous proliferation with increased mitotic activity (6 mitoses/10 high-power fields), minimal cellular atypia, and focal regressive changes with necrosis was found, and the lesion was classified as leiomyomatous neoplasia of uncertain malignant potential. Due to the increased mitotic activity, the remaining myomas were examined every 4 weeks by ultrasonography and MRI. No further growth of the remaining myomas was observed. MRI of the thorax was performed postoperatively to exclude pulmonary metastases, a known complication of sarcomas.

At 36 weeks of gestation, a cesarean section was performed to avoid onset of labor. The baby girl (2,495 g, $47 \mathrm{~cm}$, Apgar scores $8 / 8 / 9$ ) showed complete absence of the right hand directly above the wrist at delivery. The family history of the parents was negative for limb defects. The histological examination of multiple biopsy specimens from the former tumor site and the placenta showed no pathological findings. The postpartum period was uneventful.

\section{Discussion}

Complications occur in $10-40 \%$ of pregnant women with myomas and are dependent on size, location, and relation of the myoma to the placenta [3]. An increased risk of abruption has been associated with submucosal retroplacental myomas with volumes $>200 \mathrm{~cm}^{3}[2,3]$. Knowing the factors predisposing these women to complications during pregnancy, we should focus on how such complications can be prevented, thus ensuring a good pregnancy outcome. We suggest that medical therapy including analgesics, fluids, antibiotics, and prostaglandin inhibitors may be of use in patients who do not fit the high-risk group. However, in patients like our own, a timely and well-planned myomectomy should be an option offered to the patient, avoiding morbidity- and mortality-associated emergency procedures.
The literature with regard to myomectomy during pregnancy is controversial. Although Phelan [2] believes that myomatous degeneration resistant to medical therapy is an indication for surgical intervention, he limits myomectomy to pedunculated myomas with stalks no larger than $5 \mathrm{~cm}$ and avoids dissection of myomas from the uterine wall due to risks of bleeding and hysterectomy. The risk of surgery-associated spontaneous abortion has also been raised, but refuted by Mollica et al. [4] who found a $13.6 \%$ spontaneous abortion rate in conservatively treated pregnant patients with myomas and a rate of zero in the surgically treated group. The conservatively treated group also had higher premature membrane rupture and preterm labor rates, and $4.5 \%$ of them required hysterectomies after cesarean section.

Of interest in our patient, aside from her intrapregnancy myomectomy, were the histological findings. The concern for a possible malignancy had been considered with regard to the rapidity with which the patient's myoma had grown over the 4 weeks prior to surgery, even though, according to Parker et al. [5], the association between rapid growth of presumed leiomyoma and sarcoma is not substantiated. The fact that the final histology was labeled as borderline malignancy is also of interest in that it brings up the question of whether these histological findings can be considered to be within normal limits for a pregnant woman. The fact that follow-up biopsy specimens at the time of cesarean section revealed no evidence of persistent disease may support this argument.

A final point of interest meriting discussion is the unexpected limb defect found at birth. There have been a few cases described in the literature with myoma-associated fetal limb defects thought to stem from the spatial restriction caused by large fibroids [6]. Danzer et al. [7] reported the only case associating a fetal limb defect and hydrocephalus in one twin in a dichorionic, diamnionic pregnancy with a perioperative retroplacental hemorrhage possible resulting from a myomectomy performed at 10 weeks of gestation. In our patient, surgery itself is unlikely to have caused the limb defect, since it was performed at 18 weeks of gestation and since the fetal membranes were never injured. In addition, during retrospective review of the imaging studies prior to the patient's myomectomy, the fetal limb defect could be visualized, and it was most likely not prenatally diagnosed due to human error. Thus, pregnant patients with large myomas should have thorough fetal anatomical scans to exclude fetal limb abnormalities. 
In conclusion, our decision to perform a myomectomy during pregnancy was based on the fibroid size, the rapid growth, and the retroplacental location. The myoma at 18 weeks of gestation had completely filled our patient's abdomen, and further growth would most likely have induced abortion, placental abruption, or preterm labor. Therefore, a carefully planned myomectomy in high-risk pregnant patients with myomas is an appropriate lowmorbidity option which should be offered to the patient.

\section{References}

1 Katz VL, Dotters DJ, Droegemeuller W: Complications of uterine leiomyomas in pregnancy. Obstet Gynecol 1989;73:593-596.

2 Phelan JP: Myomas and pregnancy. Obstet Gynecol Clin North Am 1995;22:801-805.

3 Exacoustos C, Rosati P: Ultrasound diagnosis of uterine myomas and complications in pregnancy [see comments]. Obstet Gynecol 1993; 82:97-101.
4 Mollica G, Pittini L, Minganti E, Perri G, Pansini F: Elective uterine myomectomy in pregnant women. Clin Exp Obstet Gynecol 1996; 23:168-172.

5 Parker WH, Fu YS, Berek JS: Uterine sarcoma in patients operated on for presumed leiomyoma and rapidly growing leiomyoma. Obstet Gynecol 1994;83:414-418.
6 Graham JM, Miller ME, Stephan MJ, Smith DW: Limb reduction anomalies and early in utero limb compression. J Pediatr 1980;96: 1052-1056.

7 Danzer E, Holzgreve W, Batukan C, Miny P, Tercanli S, Hoesli I: Myomectomy during the first trimester resulting in fetal limb anomalies and hydrocephalus in a twin pregnancy. Prenat Diagn 2001;21:848-851. 